\title{
Optimal Placement of PMU with FACTS for Voltage Stability Analysis
}

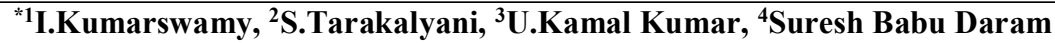

${ }^{1,3,4,}$ Department of Electrical and Electronics Engineering, Sree Vidyanikethan Engineering College, Tirupati

${ }^{2}$ Department of Electrical and Electronics Engineering, Jawaharlal Nehru Technological University, Hyderabad Email:kumarswamy04@gmail.com, tarasunder98@jntuh.ac.in,kumarkamal41@gmail.com, sureshbabudaram@gmail.com

Received: 06th December 2019, Accepted: 20th January 2020, Published: 30th April 2020

\begin{abstract}
In present scenario, the power demand has been increased briskly where there is a necessity for power system to operate within limits. As the load varies continuously, the voltage stability problems will arise. The foremost cause of voltage instability is deficiency of reactive power. The voltage stability and power transfer capability can be enhanced by compensation of reactive power. By placing Flexible AC Transmission System (FACTS) devices the performance of power system can be improved but these devices are expensive so it has to be placed at optimal location. This paper deals with the optimal placement of SVC and PMU in IEEE-14 bus system using PSAT to analyse and enrich the voltage stability margin of a power system network in real time monitoring. In real time the measurement of power system parameters such as current and voltage phasors, frequency can be effectively carried out by synchronized PMU.
\end{abstract}

\section{Keywords}

Flexible AC Transmission System (FACTS); Phasor Measurement Unit (PMU); Simulated Annealing (SA); Power System Analysis Toolbox (PSAT)

\section{Introduction}

Last twenty years across worldwide most of the power system blackouts were occurred due to the stressed power system. The major factors of stressed power system are due to load variations, over voltage and shortage of reactive power [1]. As the demand of Electrical power is increasing the generation is pacing to match but, the transmission sector is not expanding at the same rate. Concurrently, the burden on transmission system had been increased due to the deregulation and privatization of the power system network [5] [11]. Due to this the power system is exposed to its limits so the voltage instability problems are arising. As the load is varying continuously the voltage at the buses will be low, the losses will be increased simultaneously [1]. To balance the voltage at the buses the network is needed to provide compensating devices with high response speed in the field of power electronics. Currently, without using of capacitors and gate turn off thyristors (GTO) the emerging technology like FACTS had used to generate reactive power [5]. The extensive use of these devices is flexibility and increase security, increases the power transfer capability, and controls the electrical parameters like voltage, impedance, and phase angle, current and damped of oscillations. FACTS devices are used to eliminate voltage instability problems [4]. A constant monitoring of the system is required to control the FACTS devices. To estimate and monitor the voltage and power flow at each bus Phasor measurement Unit (PMU) along with state estimator and Supervisory Control and Data Acquisition (SCADA) is needed. In order to find any abnormalities had take place in the system PMU required less instance. The revelation of power system has increased by combining the PMU and FACTS devices [8]. By manual operation using FACTS devices will be delayed so real time monitoring by PMU can overcome it. To synchronize the measurement at isolated areas the PMU are installed at large number of buses. From PMU the synchrophsasors is acquired which is large data, through Wide Area Network (WAN) communication the data is transferred to Phasor Data Concentrator (PDC) using standards and protocols. By using different algorithms the operator can be analysed the synchrophasor data [7].

Phasor Measurement Unit (PMU)

In the word PMU the phasor represents a sinusoidal signal. From the Figure 1 the phase angle can be defined as the distance between the sinusoidal peak of signal and reference as time.

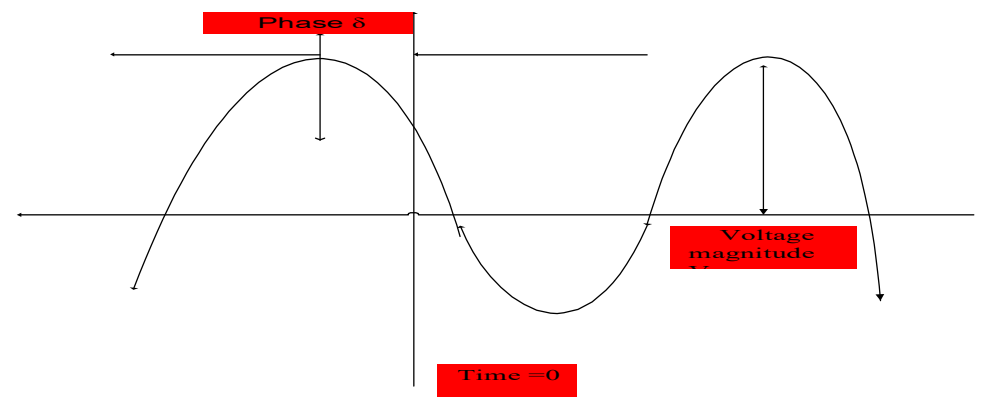




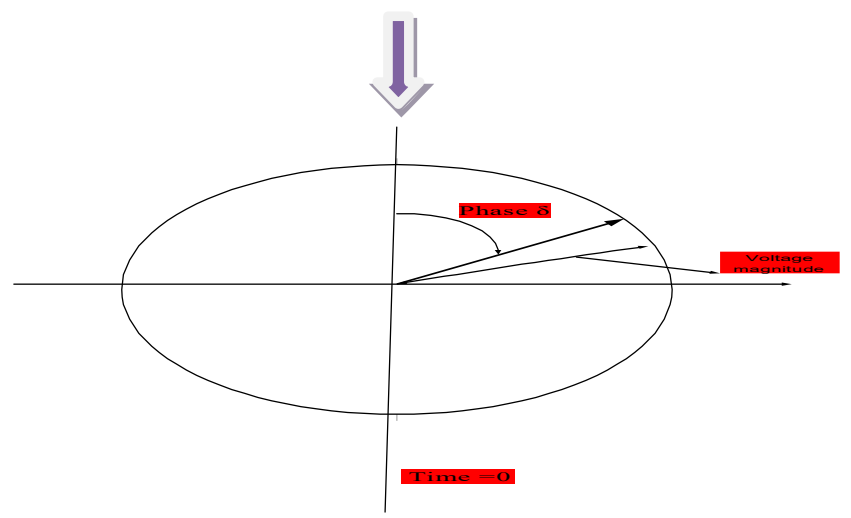

Figure 1: Sinusoidal Waveform and Phasor Representation

Table 1: Foremost Components of PMU

\begin{tabular}{|c|c|c|}
\hline S.No & Components & Function \\
\hline 1 & Analog signal & $\begin{array}{r}\text { The input to the PMU are current and voltage from } \\
\text { potential transformer and current } \\
\text { transformer }\end{array}$ \\
\hline 2 & Anti-aliasing filter & $\begin{array}{c}\text { Which is low pass filter it eliminates the } \\
\text { frequencies } \geq \text { half of nyquist rate }\end{array}$ \\
\hline 3 & $\begin{array}{c}\text { Phase locked } \\
\text { oscillator }\end{array}$ & $\begin{array}{c}\text { Stabilize the input signal } \\
\text { GPS receiver }\end{array}$ \\
\hline 5 & A/D converter & Converts analog signals to digital signals \\
\hline 6 & Micro-processor & $\begin{array}{r}\text { Using Discrete Fourier Transforms (DFT) analysis, } \\
\text { it calculates positive sequence of } \\
\text { estimated voltage and current. }\end{array}$ \\
\hline 7 & Modem & $\begin{array}{r}\text { A signal is produced and decoded to make a copy } \\
\text { of unique digital information. }\end{array}$ \\
\hline
\end{tabular}

Table1. Represents the components of PMU and its functions and the basic block representation of PMU are shown in below Figure 2.

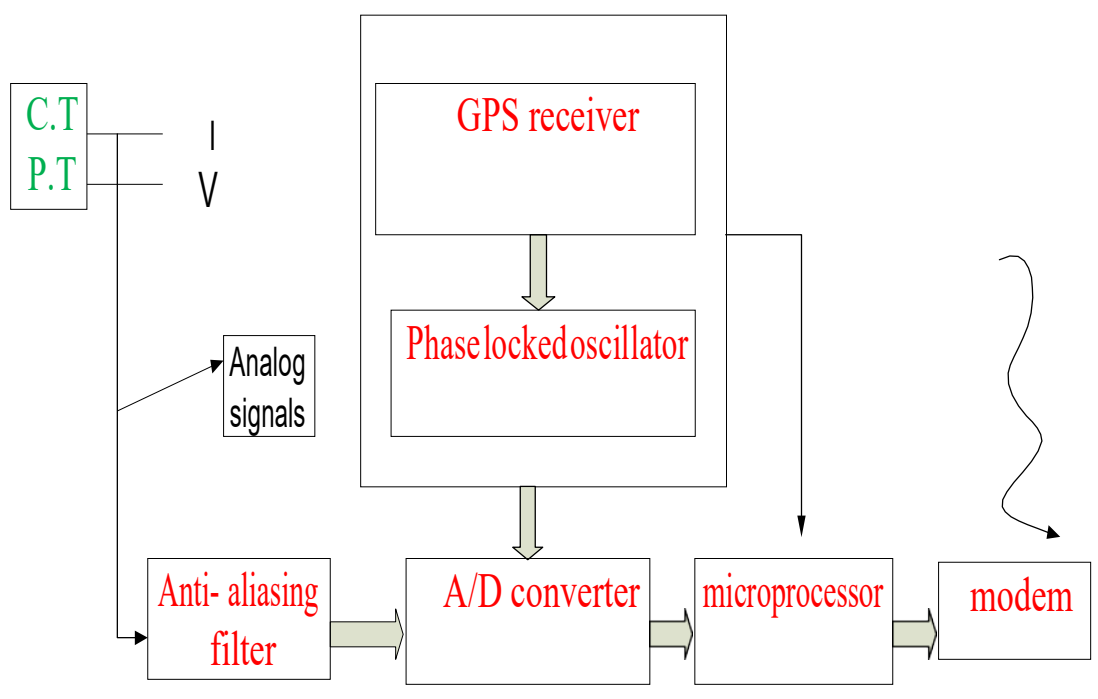

Figure 2: Pictorial Representation of PMU

If any blackouts or any major fault had occurred in the power system network, through PMU the status of the system can be known and also the sequential events can be recorded without any time delay. Based on this the power system is protected to provide time synchronizing pulse; the Global Positioning System (GPS) is used and it provides onepulse-per-second (pps). One of the advantage of this system is the installation time has been reduced and turns out to be portable, user friendly [9]. 
Mathematical Representation of PMU

$\mathrm{AC}$ waveform can be represented as

$\mathrm{X}(\mathrm{t})=\mathrm{Xm} \cos (\omega \mathrm{t}+\theta)$

Where $\mathrm{Xm}$ is the magnitude of the waveform

$\theta$ is the angle

$\omega=2 * \pi * f$

' $\mathrm{f}$ ' is the frequency

Phasor representation

$\mathrm{X}=\cos \theta+\mathrm{j} \sin \theta$

Where $\mathrm{X}$ is the RMS value of the waveform. The value depends on the time scale that is at $\mathrm{t}=0$ as shown in Fig. 1 .

\section{FACTS Controllers}

In past most of the controllers were mechanical in nature. In mechanical there are in-built problems. To overcome this FACTS controllers are designed which has more possibilities increase the power of existing line with huge conductors. FACTS devices are the new devices emerging from innovative technologies which are capable to change impedance, phase angle at particular points in the power systems. The FACTS devices have high potential to enhance the power system stability [6].

FACTS controllers are categorized in to 4

(i) Shunt device

(ii) Series device

(iii) Series-shunt device

(iv) Shunt-shunt device

Among various FACTS controllers Static VAR Compensator (SVC) is majorly used to improve the bus voltage and improves the stability of the test bus system. Around the globe greater than 700 SVC are widely used. As the SVC is a shunt connected device whose output can be varied by adjusting inductive or capacitive currents [3]. For proper operation and consumption of loads the voltage levels should be rated. Due to under voltage the performance of loads is degraded such as induction motors. Due to overvoltage the harmonics will generate and equipment gets damaged.

\section{Static Var Compensator (SVC)}

SVC consists of a TCR in similar with a bank of capacitors and SVC behaves like a shunt connected variable reactance from the operational point of view. To achieve instantaneous speed of response the SVC is enabled by firing angle control method. The Elements which may be used to make an SVC typically include as shown in Figure 3.

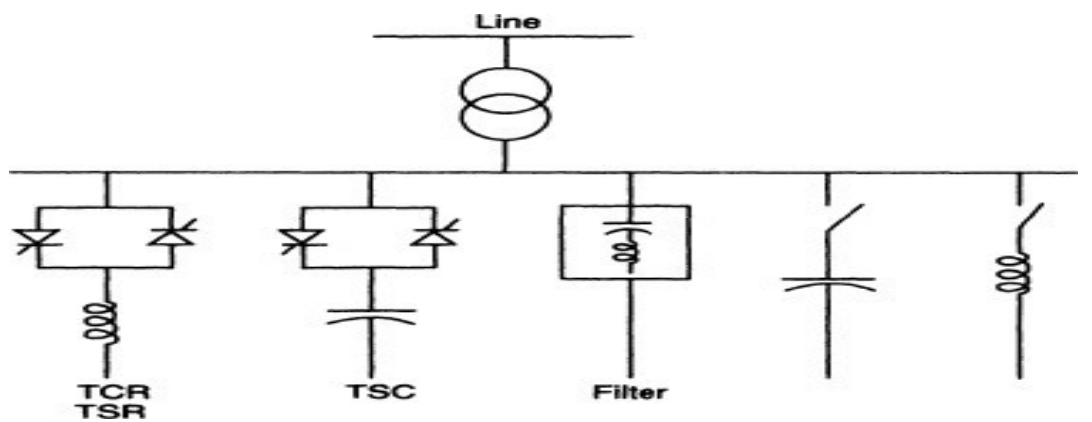

Figure 3: Schematic Diagram of SVC

As SVC has no moving parts distinct like a synchronous condenser which is a rotating electrical machine. Power factor compensation is one of the important factors of the large rotating machines such as synchronous condenser before invention of SVC. In transmission side SVC is used to regulate the grid voltage. When the load is capacitive (leading) the SVC will consume reactive power by lowering the system voltage. Consequently, when the load is inductive (lagging), the SVC will absorb reactive power by increasing the system voltage. SVC is generally placed at fast changing loads in industrial applications such as arc furnace. During the operation of thyristor controlled rectifiers (TCR) the current harmonics are unavoidable so, to eliminate harmonics the filters are necessary [1].

\section{Mathematical Analysis of SVC}

Basically two configurations are considered i.e the variable susceptance model and firing -angle model. Specifically, the variable susceptance model is considered as shown in Figure 4. 


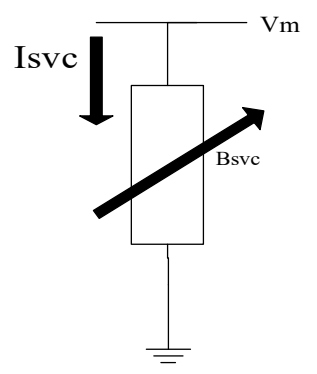

Figure 4: Susceptance Model of SVC

The voltage control can be achieved by varying $\mathrm{B}_{\mathrm{svc}}$. Either reactance limit or firing angle limits the SVC can act as adjustable reactance. Let the current (electrical charge carriers) drawn by SVC

$\mathrm{I}_{\mathrm{svc}}=\mathrm{Y} \mathrm{V} \mathrm{V}_{\mathrm{m}}$

$\mathrm{Y}=\mathrm{G}+\mathrm{jB}$ svc

In general the synchronous generator is modeled as same as SVC with Zero real power generation, and reactive power generation specified for upper and lower limits. Consequently, Based on the assumption there was absence of resistive component in SVC. Mathematically, it was represented as

$\mathrm{G}_{\mathrm{svc}}=0$

$I_{\mathrm{svc}}=j B_{\mathrm{svc}} \mathrm{V}_{\mathrm{m}}$

Where $V_{m}$ is the voltage at bus ' $m$ ' $Y$ is the admittance of the system $G$ is the conductance

$\mathrm{B}$ is the susceptance The reactive power injected at bus ' $\mathrm{m}$ ' is

$\mathrm{Qk}=-\mathrm{V}_{\mathrm{m}}^{2} \mathrm{~B}_{\mathrm{svc}}$

The susceptance value of SVC will be updated at the ending of every iteration (j). Mathematically, it represented as $\mathrm{Bsvc}=\mathrm{B}_{\mathrm{svc}}{ }^{(\mathrm{i}-1)} \cdot \mathrm{B}_{\mathrm{svc}}{ }^{(\mathrm{j}-1)}$

By varying the susceptance the voltage magnitude can be improved.

Optimal Placement of FACTS AND PMU

Optimal placement of FACTS device can be decided by the identification of weak bus. Voltage stability can be analysed by static and dynamic method .one of the proficient and simple method of static analysis is the modal analysis. By using Modal analysis the critical bus is identified [2]. The voltage magnitude of a particular bus which leads to voltage instability state is known as critical bus. The optimal placement of PMU can be decided by simulated Annealing (SA) algorithm [11].

\section{Simulated Annealing (SA)}

Depth first search and simulated annealing are the heuristics method. To deal the complex non-linear problems Kirkpatrick proposed the Simulated Annealing algorithm in 1983. SA algorithm is used for global optimization. In SA the optimization process is annealing of molten metals. In order to maintain the system from melting, the annealing was cooled down slowly and sufficiently. The solid atoms will be ordered at the time of cooling. As cooling increased beyond normal state, the system will become too cold state [9]. The temperature should not be low because to keep away the system from meta-stable state. The objective of SA algorithm is to locate global minimum and try to avoid local minimum. The SA algorithms proceed with minimum and maximum voltage and angle values, and from the load bus the maximum power is removed. The algorithm for SA is as follows:

Step1: It begins with a random initial solution, $X$, and an initial temperature $T$, which should be high enough to allow all candidate solutions to be accepted and to evaluate the objective function.

Step 2: set $\mathrm{i}=\mathrm{i}+1$ and the generated new solution $\mathrm{Xi}$ new $=\mathrm{Xi}+\mathrm{r}$ sLi where $\mathrm{r}$ is a random number. sLi should be decreased with the reduction of temperature. Evaluate Fl new $=F(X 1$ new $)$

Step 3: Choose accept or reject the move. The probability of acceptance (depending on the current temperature) if Fi new $<$ Fi-1 go to step 5 . Accept Fi as the new solution with probability

Step 4: If F I was rejected in Step 3, set Fi new - Fi-1 .Go to step 5.

Step 5: If satisfied with the current objective function value, Fi is stopped. Otherwise, adjust the temperature Tnew $=$ Tnt where $n t$ is the temperature reduction rate called cooling schedule and go to Step 2. The process is done until freezing point is reached. 


\section{Results}

From the analysis of IEEE 14 bus system the weakest bus is identified and the SVC has been placed at that bus. The IEEE 14 bus system includes 9 load buses with voltage limits, 4 generator buses with reactive power limits and voltage limits, 1 slack bus, and 4 transformers. By using PSAT software the voltage stability is analysed. From table 2 the base case voltage magnitude is represented by power flow analysis using Newton Raphson (N.R) method for 4 iterations it was converged.

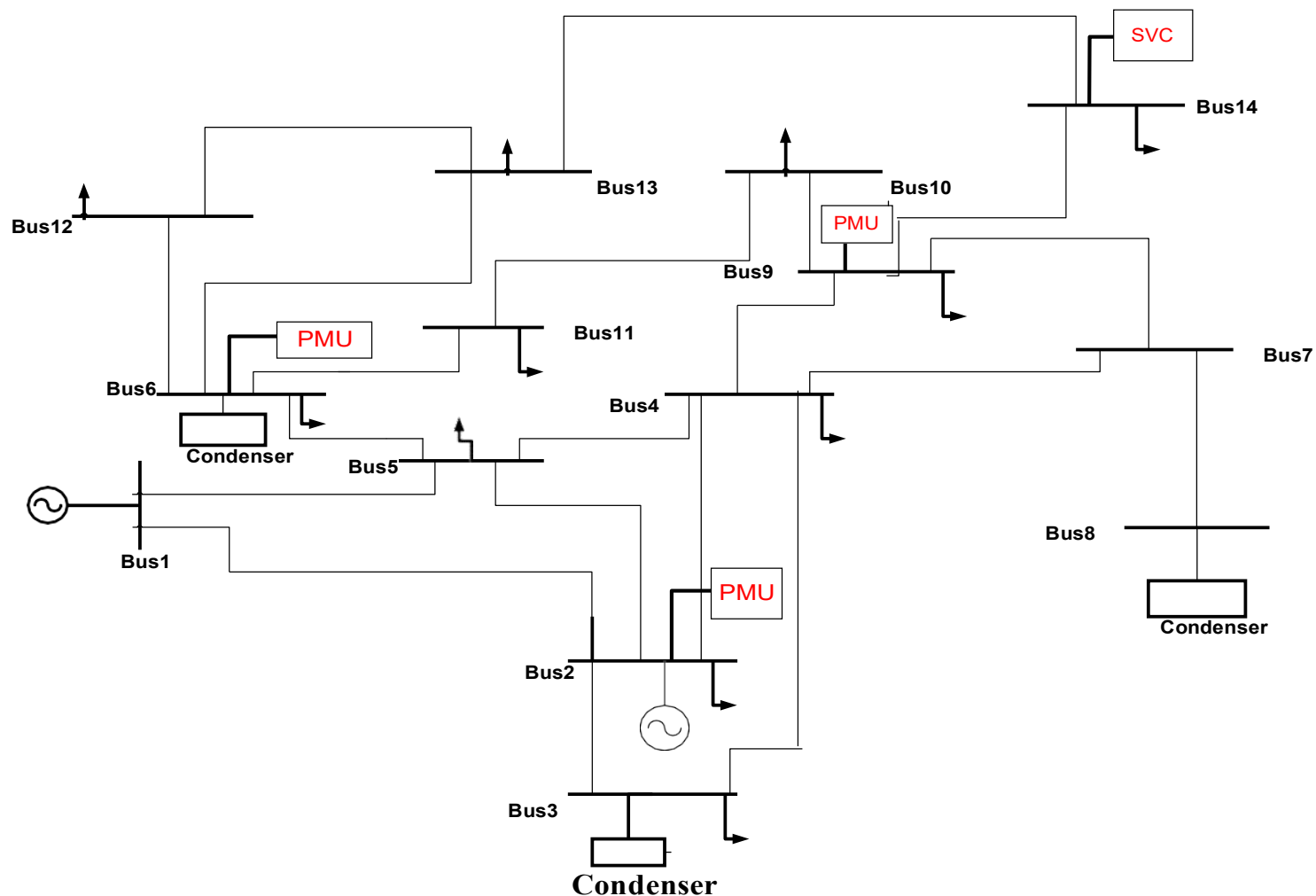

Figure 5: IEEE 14 bus test system with PMU and FACTS

Table 2: Voltage Magnitude for IEEE 14 Bus System

\begin{tabular}{|c|c|}
\hline Bus number & $\begin{array}{c}\text { Voltage magnitude } \\
\text { (P.u) }\end{array}$ \\
\hline 1 & 1.06 \\
\hline 2 & 1.045 \\
\hline 3 & 1.01 \\
\hline 4 & 1.0105 \\
\hline 5 & 1.0144 \\
\hline 6 & 1.07 \\
\hline 7 & 1.0487 \\
\hline 8 & 1.09 \\
\hline 9 & 1.0321 \\
\hline 10 & 1.0312 \\
\hline 11 & 1.0468 \\
\hline 12 & 1.0534 \\
\hline 13 & 1.0469 \\
\hline 14 & 1.0203 \\
\hline
\end{tabular}


As there is a load variation in a system there is reducement in voltage values. The load has been increased to $70 \%$ and $80 \%$ and respective voltage values are taken as shown in Table 3. To compensate this FACTS device are placed at appropriate location. By considering 80\% loading the weak bus is identified using Eigen values and its participation factor by modal analysis .From table 4 the least Eigen value is at bus number 14 and the participation factor is calculated for this critical bus. It can be analysed that the highest participation factor is the weakest bus that is bus number 14 as shown in Table 4 . The red line of P.F indicates the weak bus.

Table 3: Increment of Loading with $70 \%$ and $80 \%$

\begin{tabular}{|c|c|c|}
\hline \multicolumn{3}{|c|}{ Voltage Magnitude (P.u) } \\
\hline Bus number & $\begin{array}{c}70 \% \\
\text { loading }\end{array}$ & $\begin{array}{c}80 \% \\
\text { loading }\end{array}$ \\
\hline 1 & 1.06 & 1.06 \\
\hline 2 & 1.045 & 1.045 \\
\hline 3 & 1.01 & 1.01 \\
\hline 4 & 0.98389 & 0.979 \\
\hline 5 & 0.98947 & 0.985 \\
\hline 6 & 1.07 & 1.07 \\
\hline 7 & 1.024 & 1.0199 \\
\hline 8 & 1.09 & 1.09 \\
\hline 9 & 0.99579 & 0.98988 \\
\hline 10 & 0.99561 & 0.98988 \\
\hline 11 & 1.0261 & 1.0228 \\
\hline 12 & 1.0404 & 1.0385 \\
\hline 13 & 1.0283 & 1.0255 \\
\hline 14 & 0.97734 & 0.97055 \\
\hline
\end{tabular}

Table 4: Eigen Values and its Participation Factors

\begin{tabular}{|c|c|c|}
\hline Bus number & Eigen value & $\begin{array}{c}\text { Participation } \\
\text { Factor }\end{array}$ \\
\hline 4 & 63.0928 & 0.00867 \\
\hline 9 & 37.8522 & 0.20123 \\
\hline 7 & 21.0536 & 0.06901 \\
\hline 13 & 18.4584 & 0.03117 \\
\hline 5 & 15.6315 & 0.00434 \\
\hline 11 & 10.858 & 0.10577 \\
\hline 10 & 7.3804 & 0.23912 \\
\hline 12 & 5.3464 & 0.01735 \\
\hline 14 & 2.5257 & 0.32334 \\
\hline
\end{tabular}

The optimal location of SVC is placed at bus number 14 as shown in Table 5 . The voltage improvement/ security have been enhanced from 0.97 P.u to 0.99 P.u. By SA method the PMU with SVC are placed at various locations as shown in table 6. The voltage is enhanced by placing PMU at bus number 6, 9, and 2 as shown in Table 7. 
Table 5: Comparison of with SVC and without SVC of Voltage Magnitude

\begin{tabular}{|c|c|c|}
\hline \multicolumn{3}{|c|}{ Voltage magnitude (P.u) } \\
\hline Bus number & without placing SVC device & Placing SVC \\
\hline 1 & 1.06 & 1.06 \\
\hline 2 & 1.045 & 1.045 \\
\hline 3 & 1.01 & 1.01 \\
\hline 4 & 0.979 & 0.98384 \\
\hline 5 & 0.985 & 0.98945 \\
\hline 6 & 1.07 & 1.07 \\
\hline 7 & 1.0199 & 1.024 \\
\hline 8 & 1.09 & 1.09 \\
\hline 9 & 0.98988 & 0.99566 \\
\hline 10 & 0.98988 & 0.99545 \\
\hline 11 & 1.0228 & 1.026 \\
\hline 12 & 1.0385 & 1.0404 \\
\hline 13 & 1.0255 & 1.0283 \\
\hline 14 & 0.97055 & 0.9772 \\
\hline
\end{tabular}

Table 6: PMU Location by Simulated Annealing Method

\begin{tabular}{|c|c|c|}
\hline PMU Location & Estimated voltage & Estimated $\theta$ \\
\hline 2 & 1.0151 & -0.2792 \\
\hline 6 & 1.0312 & -0.42164 \\
\hline 9 & 1.040 & -0.40785 \\
\hline
\end{tabular}

Table 7: Enhancement of Voltage with SVC and PMU withSVC (Blue Line indicates the Placement of PMU with SVC).

\begin{tabular}{|c|c|c|}
\hline \multicolumn{2}{|c|}{ Base case SVC } & PMU with SVC \\
\hline $\begin{array}{c}\text { Bus } \\
\text { number }\end{array}$ & $\begin{array}{c}\text { Voltage magnitude } \\
\text { (P.u) }\end{array}$ & $\begin{array}{c}\text { Voltage magnitude } \\
\text { (P.u) }\end{array}$ \\
\hline 1 & 1.06 & 1.06 \\
\hline 2 & 1.045 & 1.045 \\
\hline 3 & 1.01 & 1.01 \\
\hline 4 & 0.98384 & 1 \\
\hline 5 & 0.98945 & 1.0027 \\
\hline 6 & 1.07 & 1.07 \\
\hline 7 & 1.024 & 1.052 \\
\hline 8 & 1.09 & 1.09 \\
\hline 9 & 0.99566 & 1.045 \\
\hline 10 & 0.99545 & 1.0477 \\
\hline 11 & 1.026 & 1.0439 \\
\hline 12 & 1.0404 & 1.0361 \\
\hline 13 & 1.0283 & 1.0095 \\
\hline 14 & 0.9772 & \\
\hline
\end{tabular}




\section{Conclusion}

As the power system network is stressed there will be occurrence of large disturbances such as loss of generation, contingencies and the sudden increment of load. Due to this the voltage stability problems are arising. To overcome this problems the compensation devices like FACTS are placed at appropriate location to enhance the voltage stability. Comparing to FACTS the PMU is capable for real time operation. Significantly, the synchrophasor technology improves the visualization accessible at the control centers. Large quantity of data is stored at control centers. Due to the less availability of offline and real time applications so, it is complicated to analyse that data at control centers. For better visualization the combination of PMU data with SCADA/ EMS is used. By assessment the voltage has been enhanced by placing PMU with FACTS than placing PMU.

\section{References}

1. Sarita S.Bhole, Prateek Nigam, "Improvement of voltage stability in power system by using SVC and STATCOM", International Journal of Advanced Research in Electrical, Electronics and Instrumentation Engineering (IJAREEIE), Vol.4, No.2, PP. 749-755, 2015.

2. Mitali Chakravorty, Sarmila Patra, "Voltage stability analysis using conventional methods", International Conference on Signal processing Communication Power and Embedded System (SCOPES), Paralakhemundi, India, October 3-5, 2016.

3. Hajer Jmii, Asma Meddeb, Souad Chebbi "Assessment of SVC in Improving Power system Stability”, International Science Press, Vol.9, No.38, PP.189-201, 2016.

4. Ramesh Kumari, Parveen,"Brief Study on TSCS, SSSC, SVC Facts Device", International Journal of Advanced Research in Electrical, Electronics and Instrumentation Engineering (IJAREEIE), Vol.5, N0.8, PP. 7053-7060, 2016.

5. Bindeshwar Singh, Garima Agrawal, "Enhancement of voltage profile by incorporation of SVC in power system networks by using optimal load flow method in MATLAB/ SIMULINK environments", Vol.4, PP.418-434, 2018.

6. N.M.G. Kumar, P.Venkatesh, Dr. P.Sangamewara Raju "Modeling and Analysis of SVC, TCSC, TCPAR in Power Flow Studies", International Journal of Emerging Technology and Advanced Engineering (IJETAE), Vol.3, No.1, PP. 418-425, 2013.

7. Mohd Tauseef Khan, Anwar Shahzad Siddiqui,"FACTS device Control Strategy using PMU", Elsevier, Vol.8, PP.730-732, 2016.

8. P.K Agarwal, V.K.Agrawal, Harish Rathour, "Application of PMU- Based Information in the Indian Power System", International journal of Emerging Electric Power Systems,Vol. 14, No.1, PP. 7986,2013

9. Md Kamrul Hasan Pulok, "Development of Real -Time Voltage Stability Monitoring Tool for Power System Transmission Network Using Synchrophasor Data", July 13, 2015

10. "Voltage Stability Analysis By Simulated Annealing Algorithm", International Journal of Engineering and Techniques, Vol.3, No.3, PP.115-121, 2017.

11. I kumaraswamy, B. VenkataPrasanth, S. Tarakalyani, "Role of Distributed generation for Voltage Stability Enhancement", International Journal of Current Engineering And Technology, Vol.4, No.1, PP.60-64, 2014.

12. I. kumaraswamy, W.V. Jahnavi, T.Devaraju, P.Ramesh, "An optimal power Flow (OPF) Method with Improved Voltage Stability Analysis", Proceeding of the World Congress on Engineering, London U.K, July 4-6, 2012. 